\title{
Neuroeducación y planeación didáctica en la práctica docente
}

\section{Neuroeducation and didactic planning in teaching practice}

DOI: 10.46932/sfjdv3n1-103

Received in: Jan 30st, 2021

Accepted in: Feb 1th, 2022

Mtra. Sarai Vázquez Solórzano

Maestra en Práctica Docente. Universidad Autónoma del Estado de México, México. Facultad de Ciencias de la Conducta.

E-mail: sarivazquezsolorzano@gmail.com

\section{Dr. Enrique Navarrete Sánchez}

Profesor de Tiempo Completo (PTC) Facultad de Ciencias de la Conducta de la Universidad Autónoma del Estado de México, México

E-mail: eenavarretes@gmail.com

\section{Dra. María del Carmen Farfán García}

Farfán García Profesor de Tiempo Completo (PTC) Facultad de Ciencias de la Conducta de la Universidad Autónoma del Estado de México, México

E-mail: mcfarfang@uaemex.mx

\section{Dra. Daisy González Parada}

la investigadora al frente de D.G. Educa. Centro de capacitación en Neuroeducación

E-mail: daisygonzalez3000@gmail.com

\section{RESUMEN}

En el presente artículo muestro el proceso que realicé para lograr una mejora en mi práctica docente, con base en el modelo de investigación-acción de Navarrete \& Farfán (2016). En primer lugar, reflexioné acerca de mi labor docente para detectar las áreas de mejora, para ello se inició con la pregunta ¿cómo llegué a ser docente? para tener una mirada introspectiva de mi rol como docente, además realicé un análisis de videos de clase, diario de alumnos y diario del docente, pude detectar algunas problemáticas de la práctica docente las cuales son: planeación didáctica deficiente, falta de control de grupo y baja motivación. Para dar solución a estos problemas propongo utilizar el modelo MIND (Modelo de Intervención Neuro Didáctica) para realizar una planeación didáctica y así ir mejorando el control de grupo y la motivación.

Palabras clave: Práctica docente, neuroeducación, modelo MIND, planeación didáctica, investigaciónacción.

\begin{abstract}
In this article I show the process I carried out to achieve an improvement in my teaching practice, based on the action research model of Navarrete \& Farfán (2016). First of all, I reflected on my teaching work to detect areas for improvement, for this I started with the question "How did I become a teacher?" to have an introspective look at my role as a teacher, I also conducted an analysis of class videos, students' diary and teacher's diary, I could detect some problems of teaching practice which are: deficient didactic planning, lack of group control and low motivation. To solve these problems I propose to use the MIND
\end{abstract}


model (Model of Neuro Didactic Intervention) to make a didactic planning and thus improve group control and motivation.

Keywords: Teaching practice, neuroeducation, MIND model, didactic planning, action research.

\section{INTRODUCCIÓN}

En este documento se presenta la aplicación del modelo de investigación-acción, en lo sucesivo IA diseñado por Navarrete \& Farfán, (2016), en éste, se realiza primero un análisis de la práctica docente, para este caso la práctica docente analizada fue la mía, el lugar de trabajo el Telebachillerato Comunitario Núm. 02 San Pedro la Hortaliza, en el municipio de Almoloya de Juárez, en un grupo de 25 alumnos de $2^{\circ}$ semestre, en el área de Ciencias Experimentales.

Para detectar las áreas con problemática o con oportunidad de mejorar se utilizaron tres instrumentos: diario reflexivo del alumno, diario del docente y videos de clase, después de realizar el análisis pertinente de cada uno, detecté que existe una planeación didáctica deficiente, falta de control de grupo y baja motivación.

Tal como lo dice el modelo de I-A que se está aplicando, propongo un modelo de intervención educativa que surge a partir de una pregunta inclusiva y un supuesto de acción, en este caso se propone usar el Modelo de Intervención Neuro Didáctica MIND para mejorar o solucionar las problemáticas detectadas.

\section{DESCRIPCIÓN DEL MÉTODO}

\subsection{MODELO DE INVESTIGACIÓN-ACCIÓN.}

Para empezar a definir lo que es la investigación-acción retomaré algunos conceptos de diversos autores citados en Navarrete \& Farfán, (2016), en la Tabla 1. Diversos conceptos de Investigación-acción.

Tabla 1. Diversos conceptos de Investigación-acción.

\begin{tabular}{|l|l|}
\hline Lewin (1946) & $\begin{array}{l}\text { “...una forma de cuestionamiento autorreflexivo, llevada a cabo por los propios participantes } \\
\text { en determinadas ocasiones con la finalidad de mejorar la racionalidad y la justicia de } \\
\text { situaciones de la propia práctica social educativa, con el objetivo también de mejorar el } \\
\text { conocimiento de dicha práctica y sobre las situaciones en las que la acción se lleva a cabo". }\end{array}$ \\
\hline Moser (1978) & "...el conocimiento práctico no es el objetivo de la investigación-acción sino el comienzo". \\
\hline Bartolomé (1986) & $\begin{array}{l}\text { Es un proceso de reflexión en el que investigación-acción y formación de los profesionales en } \\
\text { ciencias sociales, acerca de su propia práctica, puede realizarse de manera individual o en } \\
\text { equipo, con o sin un facilitador externo. }\end{array}$ \\
\hline Kemmis (1989) & $\begin{array}{l}\text { Lo concibe como ciencia crítica ya que implica un proceso de autorreflexión, llevada a cabo } \\
\text { por los participantes, sean docentes, alumnos o autoridades, con el objetivo de mejorar las } \\
\text { prácticas sociales o educativas. }\end{array}$ \\
\hline Elliot (1993) & $\begin{array}{l}\text { La define a partir del estudio de situaciones sociales con el propósito de mejorar la calidad de } \\
\text { la acción dentro de éstas, la interpreta como la reflexión de acciones humanas y situaciones } \\
\text { sociales vividas por los profesores, tienen como objetivo ampliar la comprensión o diagnóstico } \\
\text { de los problemas prácticos de los docentes. }\end{array}$ \\
\hline
\end{tabular}


Como se observa, la investigación-acción es un proceso de autorreflexión e indagación sistemático que nos permite identificar las áreas en las que podemos hacer cambios para lograr mejoras. Es importante tener presentes las principales características de este modelo, estas se encuentran de manera general en el gráfico 1, basado en Elliot, (2010).

Gráfico 1. Principales características del Modelo de Investigación-acción.

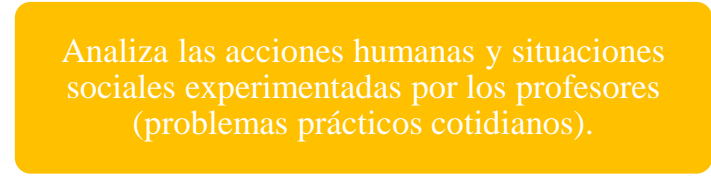

Su propósito es profundizar la comprensión del profesor (diagnóstico), de su problema.

Adopta una postura teórica para conseguir una compresnsión más profunda de un problema práctico.
Utiliza estudios de caso, lo que proporciona una

teoría naturalista presentada de forma narrativa

en lugar de una teoría formal enunciada de

forma proporcional.

Considera la situación desde el punto de vista de los participantes, por lo que se describe en el lenguaje de los mismos.

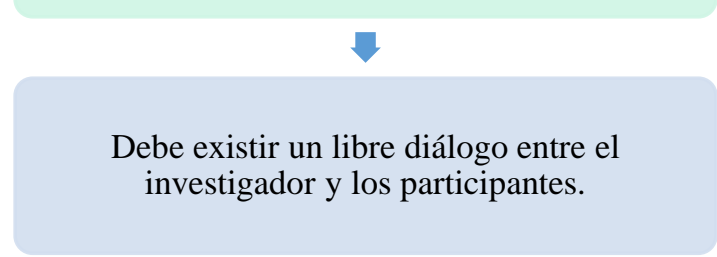

Como se puede observar en las características del modelo, el profesor es parte de la investigación, él está incluido en el objeto de estudio como el actor principal y en cada una de las etapas del desarrollo del modelo se ve involucrado y responsabilizado de llevar a cabo las acciones correspondientes. A continuación se mencionan de forma resumida las 5 etapas del modelo según Elliot (2005) citado en Navarrete \& Farfán, (2016).

1.- Problematización: es detectar las inconsistencias entre lo que se busca y lo que en realidad se está logrando.

2.- Diagnóstico: recopilación de información de las personas involucradas.

3.- Diseño de una propuesta de cambio: a través de una reflexión prospectiva, proponer cambios para lograr la mejora.

4.- Aplicación de la propuesta: se lleva a cabo la propuesta de acción, por las personas involucradas.

5.- Evaluación: las evaluaciones se realizan de manera continua durante y al final del proceso de investigación ya que pueden surgir cambios durante todo el proceso. 


\subsection{MODELO NAVARRETE, FARFÁN LABASTIDA Y MORALES}

Hablando ahora de manera específica del Modelo Navarrete et al, consultado en Navarrete \& Farfán, (2016), que es el modelo que estoy trabajando para esta investigación, se presenta el gráfico 2 donde se incluyen todos los pasos a seguir en el modelo.

Gráfico 2. Modelo Navarrete, Farfán Labastida y Morales, (2016).

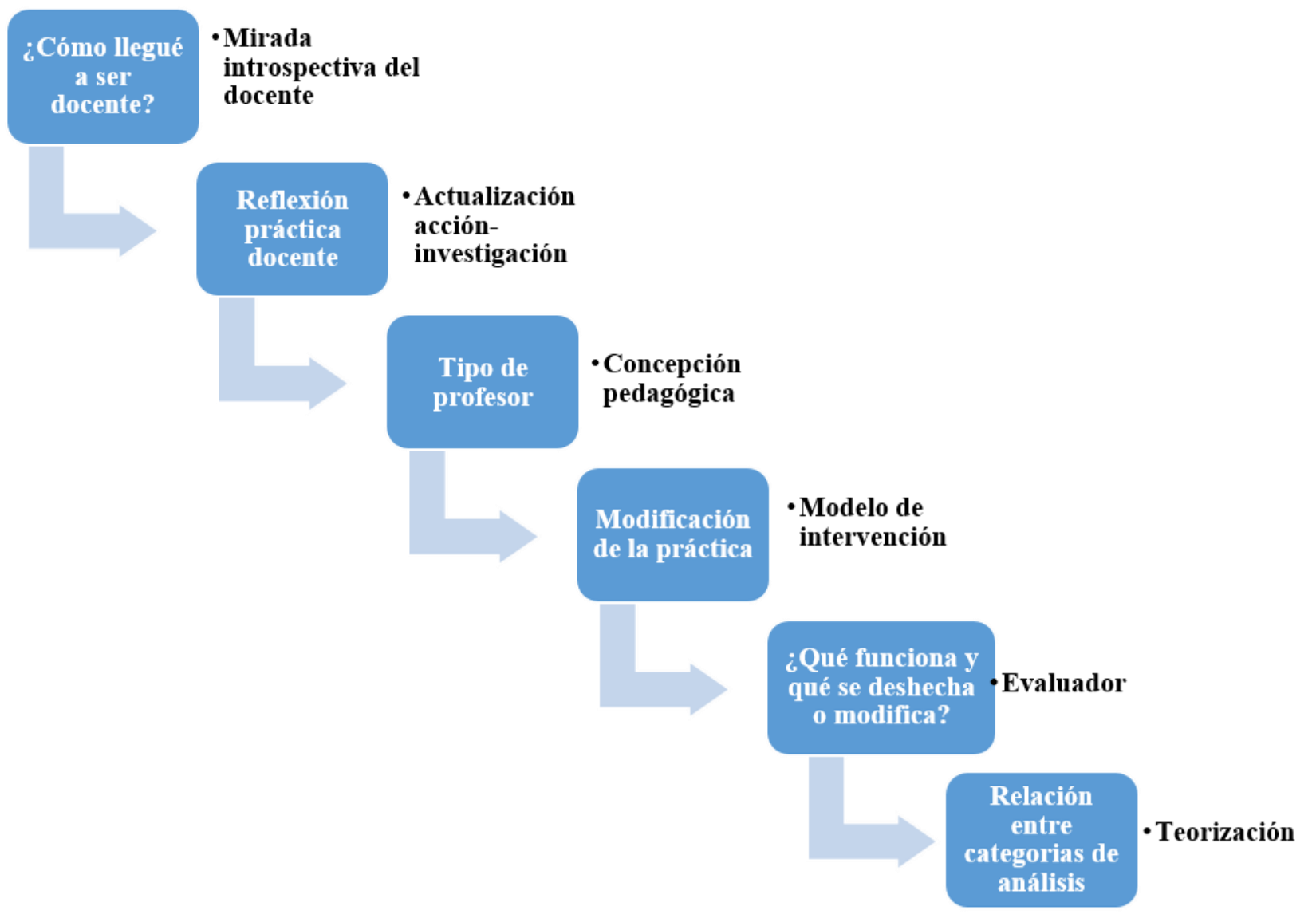

Cómo se observa en el gráfico 2, el modelo inicia con el ciclo que se denomina la mirada introspectiva del docente, en ello se reflexiona acerca de cómo se llegó a la docencia y se consideran 7 dimensiones:

1.- Dimensión personal (analizar el pasado, resignificar el presente y construir el futuro, enlazando esto con la vida profesional)

2.- Dimensión institucional (considera la manera en que la escuela influye en la práctica docente)

3.- Dimensión interpersonal (considera también las relaciones con alumnos, profesores, padres de familia, etc.)

4.- Dimensión social (condiciones heterogéneas de tipo cultural y socioeconómico del entorno)

5.- Dimensión didáctica (enseñanza e interacción de los alumnos con el saber) 


\section{6.- Dimensión valoral (valores)}

7.- Relación pedagógica (síntesis de las dimensiones anteriores)

Al observar las dimensiones de esta etapa del modelo se puede decir que la reflexión personal que el docente hace lo lleva mucho más allá de pensar solo en él como profesionista, considera todo el entorno y contexto de la vida del docente.

En el segundo ciclo se espera que el docente se vea a si mismo articulando la acción con la investigación, esto es que incorpore estrategias metodológicas como la observación participante, diarios reflexivos del docente y alumnos, videos de clase, en esta investigación solo se ocuparon estos tres instrumentos pero se pueden incluir más como entrevistas, cuestionarios y otros.

Como es de esperarse y que además es mencionado por Rosas (2003) citado en Navarrete \& Farfán, (2016), el segundo ciclo tiene gran incidencia para el tercero, la concepción pedagógica, ya que el uso de las estrategias del ciclo 2 llevan al docente a verse como en realidad es, le permite apreciar su papel desde diversos ángulos y aspectos que desde la cotidianidad de su práctica no se tienen presentes, por lo tanto el docente es capaz de detectar las habilidades y prácticas propias que posee y que caracterizan su estilo de enseñanza, es decir, puede traer a la consciencia sus fortalezas y debilidades.

Para el ciclo 4 se elige una base teórica que va permitir llevar la verdadera transformación de la práctica docente, es donde se diseña un modelo de intervención que suponemos va modificarla, este modelo por supuesto que debe contener aspectos fundamentales tales como cuándo, dónde, cómo se va desarrollar, durante cuánto tiempo, pros y contras de cada etapa, objetivos del modelo, recursos con los que se cuenta, entre otros aspectos.

Como todo proceso que se aplica, el modelo de intervención diseñado para la modificación de la práctica, debe ser sometido a un ciclo de evaluación, el objetivo es analizar lo que funcionó y cambiar lo que no para conseguir así la mejora de la práctica docente.

Para el último ciclo, teorización, el docente con base en la evaluación puede plantear un nuevo modelo de intervención con las modificaciones pertinentes o puede iniciar nuevamente el proceso en otro aspecto de la práctica que no había considerado, este ciclo se puede considerar como la síntesis del modelo de investigación-acción.

Como se observa, el modelo de investigación-acción es un modelo en forma de espiral, es decir, que no tiene un final, ya que cada vez se puede iniciar un nuevo ciclo para modificar la práctica docente en otros aspectos que se vayan descubriendo que tienen deficiencias y así poder mejorarlas. 


\subsection{MODELO DE INTERVENCIÓN NEURO PEDAGÓGICA MIND}

Como se mencionó al principio de este artículo, aquí se presenta solo un avance de la aplicación del modelo de investigación-acción, actualmente se encuentra en el ciclo de modificación de la práctica, es decir la etapa dónde se diseña un modelo de intervención pedagógica que permita hacer cambios en la práctica docente.

En el gráfico 3 se presenta un resumen de los ciclos que ya se realizaron del modelo de investigación-acción y los resultados que hubo en cada uno de los ciclos.

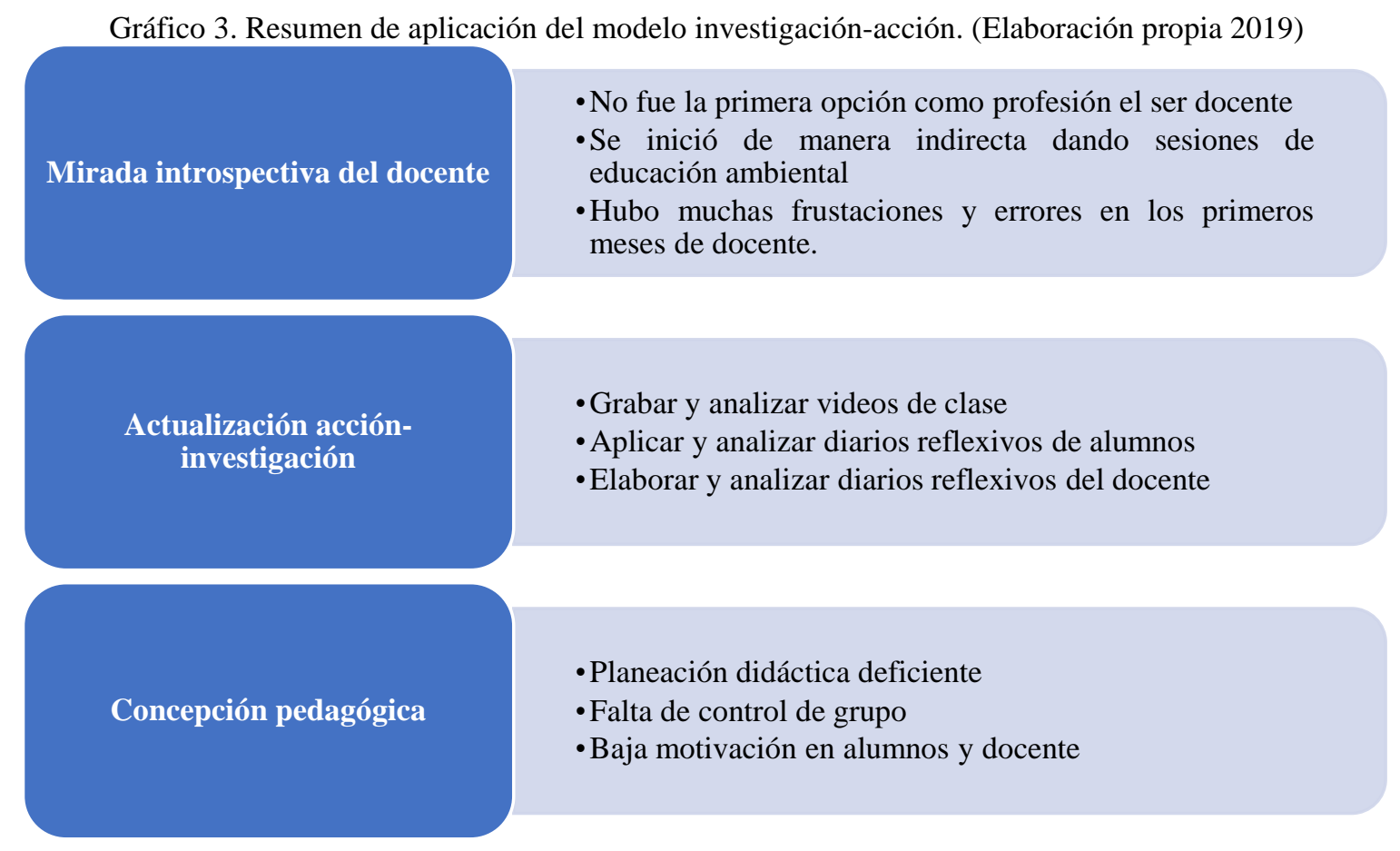

Los tres ciclos anteriores han servido como base para obtener información y así, para el ciclo 4, plantear la siguiente pregunta inclusiva: ¿Qué impacto tiene una planeación didáctica a partir del modelo MIND, para crear un ambiente de enseñanza que fortalezca la motivación docente y de los alumnos, y así lograr el desarrollo de competencias útiles en la vida cotidiana de los estudiantes?

La pregunta anterior dio como resultado el siguiente supuesto de acción: A partir de que realice la planeación y aplicación de secuencias didácticas basadas en el Modelo de Intervención Neuro Didáctico MIND, estaré promoviendo un ambiente de enseñanza-aprendizaje óptimo para la motivación y el control del grupo durante mi práctica docente.

Por lo tanto, a continuación se describe qué es y en qué consiste el modelo MIND que será la base para tomar acciones que modifican la práctica docente y así poder mejorar las tres problemáticas detectadas en los ciclos anteriores del modelo de investigación-acción. 
El modelo, por supuesto tiene su base en el funcionamiento del cerebro, es por eso que conocer de forma general el funcionamiento del mismo resulta esencial para entenderlo.

El cerebro es parte de un sistema completo, que en conjunto trabaja para recibir y procesar la información que a él llega, este sistema es el sistema nervioso.

El Sistema Nervioso (SN) está constituido por el Sistema Nervioso Central (SNC) conformado a su vez por la médula espinal y el encéfalo, y el Sistema Nervioso Periférico (SNP) que está constituido por los nervios (axones) craneales y espinales (Diamond, Scheibel, \& Elson, 2014). El cerebro tiene billones de neuronas, éstas son bloques constructivos celulares básicos del encéfalo, ellas reciben los datos de otras neuronas a través de las dendritas y según el patrón o la fuerza de estos, emiten o no un impulso nervioso como dato de salida (Seth, 2015).

Las neuronas presentan características morfológicas típicas que sustentan sus funciones: un cuerpo celular central llamado soma, una o varias prolongaciones cortas que transmiten impulsos, denominadas dendritas; y una única prolongación larga que se denomina axón, este conduce los impulsos o la información, desde el soma hacia las dendritas de otras neuronas.

Las neuronas se comunican entre sí por medio de la denominada sinapsis. Cada unión sináptica está compuesta por una terminal presináptica, que conduce un impulso nervioso a una parte de otra neurona, estructura postsináptica, que recibe el estímulo en la sinapsis, dicho estímulo debe atravesar un estrecho espacio, llamado hendidura o espacio sináptico, ya que las neuronas nunca se tocan entre sí, esto lo podemos observar en la ilustración 1.

Ilustración 1. Sinapsis neuronal. Recuperada de https://es.khanacademy.org/science/biology/human-biology/neuron-nervoussystem/a/the-synapse 03/12/2019

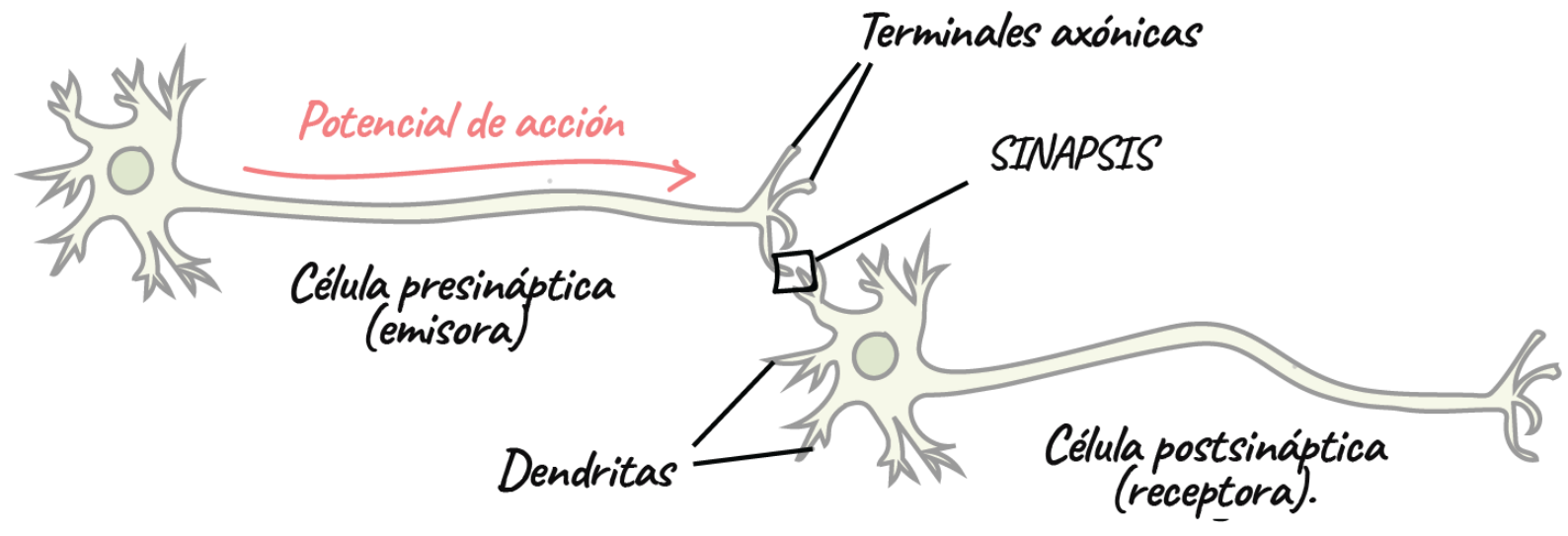

Los neurotransmisores son sustancias químicas que transmiten información de una neurona a otra en la mayoría de los procesos de comunicación que se establecen entre ellas (Braidot, 2015). Para crear un ambiente de enseñanza se van a considerar solo la dopamina, la noradrenalina y la serotonina. 
"Cada vez que aprendemos algo nuevo, se forma una red neuronal o red hebbiana" (Fernández Coto, 2014).

El principio de Hebb (científico escocés considerado el fundador de la biopsicología) afirma que “...cuando un axón de la célula A está lo suficientemente cerca de una célula B como para excitarla, y participa repetida o persistentemente en su disparo, se produce algún proceso de crecimiento o cambio metabólico en una o en ambas células, de modo que la eficacia de A, como una de las células que hacen disparar a B, aumenta".

Considerando a Fernández Coto, puedo decir que una red hebbiana es el soporte neural del aprendizaje, ya que son neuronas unidas en un circuito específico y este puede construirse, modificarse, eliminarse o potenciarse.

La neuroplasticidad adapta las neuronas y las redes neuronales al cambio en el ambiente sensorial, para Fernández Coto, nuestro cerebro se modifica de manera constante por la interacción con el medio y para Braidot, la neuroplasticidad está estrechamente ligada a cómo y por qué se modifica nuestro cerebro a medida que vamos creciendo; por lo tanto puedo decir que la neuroplasticidad tiene que ver con la forma en que las redes hebbianas se "arman y desarman" al ir aprendiendo y desarrollando conductas al estar en contacto con nuestro entorno.

Según Fernández Coto, el aprendizaje hebbiano es la base de la neuroplasticidad, si se forman redes es una neuroplasticidad positiva y se produce cada vez que aprendemos algo; por otra parte está la neuroplasticidad negativa, que se produce cuando no usamos lo que aprendemos, se va debilitando la red hasta desarmarse.

Considerando esto, para el aprendizaje, es preciso tener presente que “...si durante el proceso existe un fuerte componente emocional, si el estímulo se repitió varias veces de distintas formas, la red se consolidará por lo que el aprendizaje irá a la memoria a largo plazo, de lo contrario la red terminará desapareciendo y esas neuronas se van utilizar para formar otras redes que el cerebro considere más útil, a este proceso se le conoce como robo hebbiano" (Fernández Coto, 2014).

\subsection{MODELO MIND}

El objetivo principal de este modelo es que se pueda realizar una planeación didáctica que permita crear un ambiente de enseñanza basado en el funcionamiento del cerebro, es decir que las actividades que se incluyan para cada clase, estén diseñadas de forma en la que el cerebro trabaja de manera más natural y eficiente. Para lograrlo el MIND considera los aspectos que se muestran en el Gráfico 4. 
Gráfico 4. Modelo MIND. (Elaboración propia, 2020)

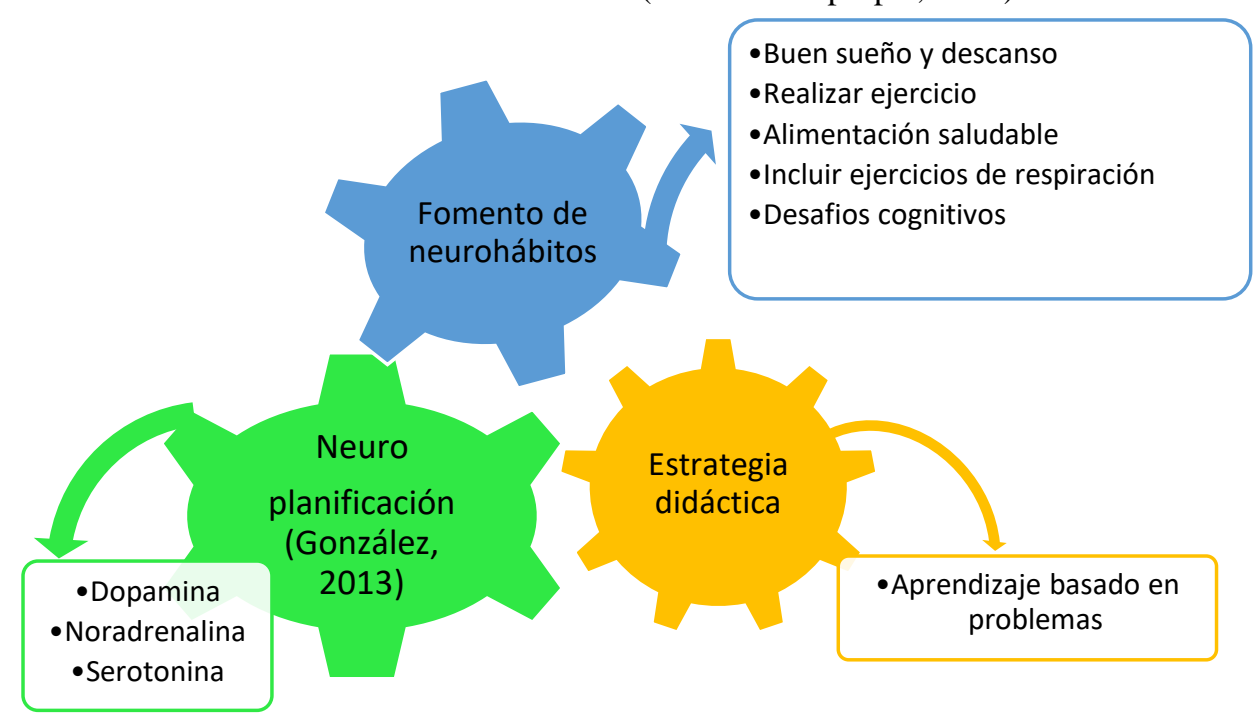

El modelo tiene 3 componentes, el primero está enfocado en fomentar hábitos en los alumnos lo que les va permitir tener una mejora en la calidad de vida y por supuesto un cerebro más saludable, apto para el aprendizaje, para ello será necesario que los alumnos conozcan el funcionamiento del cerebro y cómo usar esa información para mejorar su desempeño estudiantil, además, es muy importante considerar que la etapa en la que se encuentran los estudiantes de nivel medio superior (la adolescencia), implica procesos cerebrales muy específicos y que cuándo el alumno conoce lo que le está ocurriendo puede tener una mayor comprensión de los procesos y vivir de una manera consciente esta etapa.

Para este componente del modelo he diseñado un cuestionario que me va permitir tener información acerca de los hábitos y el estilo de vida de los estudiantes, además de que ellos van a tener presente y de manera consciente esta información, el objetivo principal de este componente es tener un diagnóstico y a partir de esto realizar actividades que fomenten hábitos de vida que aporten a tener un cuerpo-cerebro-mente saludables, para ir valorando el avance de este componente, propongo utilizar un semanario en el que cada alumno lleva un registro de las actividades que realizó y qué cambios o aportes pueden notar en su estilo de vida.

El segundo componente está basado en el modelo de neuroplanificación de González Parada (2013), una vez que los alumnos tienen conocimiento de cómo funciona su cerebro, podemos empezar a usar esa información a favor del aprendizaje, para ello se incluye en la planeación de clase actividades que estimulen la secreción de los neurotransmisores mencionados en el modelo de neuroplanificación.

El tercer componente del MIND es la estrategia de Aprendizaje Basado en Problemas (ABP), como se menciona en el marco teórico, en esta metodología se plantea un problema y se siguen una serie de pasos para poder resolverla, se eligió debido a que una de las funciones principales del cerebro es 
enfrentarse a problemas y resolverlos, intuitivamente, con el fin de mantenernos vivos, por lo tanto, es un camino que el cerebro conoce perfectamente, por decirlo de algún modo.

Para la aplicación del MIND, utilicé un instrumento de planeación de clase, éste se encuentra organizado por sesiones y se incluyen los elementos de neuroeducación retomados del modelo de neuroplanificación de González, (2013), además, a lo largo de los temas y actividades de las sesiones se abordarón temas del funcionamiento del cerebro y cómo mantenerlo saludable, también se utilizaron recursos como videos, audios, fotografías, incluso memes y uso de redes sociales para mantener al cerebro motivado y activo, ya que la novedad siempre hace que el cerebro se mantenga atento.

El plan de clase contiene tres secciones: inicio, desarrollo y cierre, en el inicio se incluyen actividades que estimulen la secreción de dopamina, es decir cosas que hagan que los alumnos se sientan con alegría y de hecho que sonrían, se pueden utilizar dinámicas, historias graciosas, juegos, canciones, entre muchas otras, el tiempo para este apartado es de 10 minutos aproximadamente, después viene la sección de actividades de desarrollo, que puede durar entre 30 y 40 minutos, aquí se considera la secreción de noradrenalina, es decir acciones, actividades que hagan que los alumnos se muevan, lean, escriban y no solo escuchen al profesor, estas actividades van a realizarse en grupos de 3 o 4 alumnos, dependiendo el tamaño del grupo, tal como lo considera el ABP.

A partir de esta sección y hasta la fase de cierre se debe promover la secreción de serotonina, esto se logra con el reconocimiento, es decir, comunicar a los estudiantes que lo están haciendo bien, o si es necesario corregir, hacerlo de una forma en la que el error no sea el centro, sino la oportunidad de mejorar y resaltar el esfuerzo que están realizando.

Para evaluar el modelo utilicé varios instrumentos, los videos de clase, diarios de alumnos y docente (que se utilizaron en la fase de diagnóstico de ésta investigación), además incluí una lista de cotejo, que permitió verificar que a lo largo de las clases planeadas se encuentran elementos de neuroeducación, se sugiere que el instrumento sea aplicado por parte del docente y por un experto en neuroeducación de preferencia aplicarlo al analizar los videos de clase.

\section{COMENTARIOS FINALES}

Como se hizo mención a lo largo de este escrito, la I-A es una espiral sin fin que puede reiniciarse al terminar un ciclo de investigación; e incluso, puede regresar a ese mismo ciclo con un nuevo enfoque o con una problemática detectada durante la investigación del mismo, por ello solo se hizo un cierre parcial de la espiral de I-A que se inició.

Las reflexiones de la docente después de aplicar el MIND fueron las siguientes: 


\section{1 "SER UNA DOCENTE REFLEXIVA".}

Este cambio es uno de los más significativos en este proceso de investigación, ya que voltear a ver la labor como docente de una forma crítica, ha sido un proceso difícil; no solo por ver que hay cosas en las que había errores, sino también aprender a reconocer y rescatar las cosas que se han hecho bien y que han funcionado.

Aceptar el equivocarse y perder el miedo a hacerlo, es una de las cosas que dejado marca en este proceso de I-A, ser capaz de observarse como una docente en construcción y mejora cada día, soltar el peso de creer que deber ser perfecta para dar un buen ejemplo y mantener una actitud reflexiva constante para poder aprovechar cada oportunidad de mejora.

\subsection{APRENDER CÓMO SE APRENDE DESDE EL CEREBRO.}

Otro de los impactos más significativos de esta investigación fue haber iniciado el proceso de aprendizaje sobre neuroeducación; conocer qué pasa en el cerebro humano, cómo funciona, entender por qué reacciona a ciertos estímulos y poder aprovecharlos, ha sido una cosa maravillosa; como persona pero sobre todo como docente.

Conocer y aplicar la neuroeducación permitió tener un nuevo enfoque y una perspectiva, de la docente que se quiere ser; es decir, seguir creyendo que es importante que los alumnos sean responsables de su aprendizaje, ya que es un proceso personal, pero no se trata de solo ceder esa responsabilidad, sino de propiciar ambientes de enseñanza que sean amigables con los procesos cerebrales, acompañándolos en el proceso y ser una guía que se lo pueda facilitar.

\subsection{SER RESILIENTE, SER DOCENTE.}

Durante todo el proceso de I-A hubo cambos de escuela, de alumnos, de la clase que se impartía e incluso de modalidad por la pandemia, todos esto dio la oportunidad de ver que en la situación que sea se sigue siendo docente, con nuevos escenarios y problemas, pero con el mismo fin que es propiciar el aprendizaje de los alumnos, lo que hizo ver que un docente en todo momento debe ser resiliente; además, ahora se tiene una nueva forma de observar y analizar las problemáticas o retos que enfrentar, con una metodología para hacerlo de manera eficiente, la I-A.

Los resultados con los alumnos también tuvieron valor significativo pero en este arículo no se hará mención de ellos, en general se puede decir que hubo cambios beneficiosos en el proceso de aprendizaje y sobre todo en el de enseñanza. 


\section{REFERENCIAS}

Braidot, N. (2015). Cómo funciona tu cerebro. Para dummies. Buenos Aires: PAFP.

Diamond, M., Scheibel, A., \& Elson, L. (2014). El cerebro humano. España: Ariel.

Elliot, J. (2010). La investigación-acción. España: Morata.

Fernández Coto, R. (2014). Cerebrando el aprendizaje. Buenos Aires: Bonum.

González Parada, D. (2013). Neuroplanificación. Modelo de Planificación del proceso de aprendizaje basado en neuroeducación. Valparaíso, Chile.

Ibarra González, K. P., \& Eccius Wellmann, C. C. (2014). Canales de aprendizaje y su vinculación con los resultados de un examen de ubicación de. Revista Intercontinental de Psicología y Educación, 16(1), $135-151$.

Jensen, E. (2010). Cerebro y aprendizaje. Competencias e implicaciones educativas. Madrid: Narcea, S. A. de ediciones.

Navarrete Sánchez, E., \& Farfán García, M. (2016). Investigación Acción. De la reflexión a la práctica educativa. Toluca de Lerdo: Colofón.

O’Brien, L. (07 de 2016). pnliafi. Obtenido de http://pnliafi.com.ar/wp-content/uploads/2016/07/testcanal-de-aprendizaje-de-preferencia.pdf

Schunk, D. H. (2012). Teorías del aprendizaje. Una perspectiva educativa (Sexta edición ed.). México: Pearson.

Seth, A. (2015). 50 temas fascinantes de la neurociencia para entender nuestro cerebro. China: Blume. 